\title{
Analysis of interpeak latencies of brainstem auditory evoked potential waveforms during microvascular decompression of cranial nerve VII for hemifacial spasm
}

\author{
Parthasarathy D. Thirumala, M.D., M.S., ${ }^{1,3}$ Preethi Ilangovan, M.B.B.S., 1 \\ Miguel Habeych, M.D., M.P.H., ${ }^{1}$ Donald J. Crammond, Ph.D., 1 \\ and Jeffrey Balzer, Ph.D. ${ }^{1,2}$ \\ Departments of ${ }^{1}$ Neurological Surgery, ${ }^{2}$ Neuroscience, and ${ }^{3}$ Neurology, University of Pittsburgh Medical \\ Center, Pittsburgh, Pennsylvania
}

\begin{abstract}
Object. Microvascular decompression (MVD) of the facial nerve is an effective treatment for patients with hemifacial spasm. Intraoperative monitoring of brainstem auditory evoked potentials (BAEPs) during MVD can reduce the incidence of hearing loss. In this study the authors' goal was to evaluate changes in interpeak latencies (IPLs) of Waves I-V, Waves III-V, and Waves I-III of BAEP Waveforms I, III, and V during MVD and correlate them with postoperative hearing loss. To date, no such study has been performed. Hearing loss is defined as nonuseful hearing (Class C/D), which is a pure tone average of more than $50 \mathrm{~dB}$ and/or speech discrimination score of less than $50 \%$.

Methods. The authors performed a retrospective analysis of IPLs of BAEPs in 93 patients who underwent intraoperative BAEP monitoring during MVD. Patients who did not have hearing loss were in Class A/B and those who had hearing loss were in Class C/D.

Results. Binary logistic regression analysis of independent IPL variables was performed. A maximum change in IPLs of Waves I-III and Waves I-V and on-skin change in IPLs of Waves I-V increases the odds of hearing loss. However, on adjusting the same variables for loss of response, change in IPLs did not increase the odds of hearing loss.

Conclusions. Changes in IPL measurements did not increase the odds of postoperative hearing loss. This information might be helpful in evaluating the value of IPLs as alarm criteria during MVD to prevent hearing loss. (http://thejns.org/doi/abs/10.3171/2012.12.FOCUS12393)
\end{abstract}

\author{
KeY WoRdS - brainstem auditory evoked potential - hemifacial spasm • \\ microvascular decompression • interpeak latency • \\ intraoperative neurophysiological monitoring
}

$\mathrm{H}$ EMIFACIAL spasm is due to neurovascular compression at the root exit zone of the facial nerve $(\mathrm{CN}$ VII). This condition can be effectively treated by MVD of CN VII. ${ }^{1,5,8,17}$ An infrequent but significant risk of MVD is hearing loss caused by damage to the vestibulocochlear nerve (CN VIII). ${ }^{1,11,17}$ Intraoperative neurophysiological monitoring of BAEPs during MVD has reduced the incidence of this complication to around 2.3\%-12.3\% by alerting the surgeons to potentially reversible situations. ${ }^{1,13,15,19,21}$ Mechanisms of damage to CN VIII include

Abbreviations used in this paper: BAEP $=$ brainstem auditory evoked potential; $\mathrm{CN}=$ cranial nerve; $\mathrm{HFS}=$ hemifacial spasm; IPL = interpeak latency; MVD = microvascular decompression; PTA = pure tone average; $\mathrm{SDS}=$ speech discrimination score. retraction of the cerebellum resulting in traction on $\mathrm{CN}$ VIII, manipulation of its blood supply, direct mechanical/ thermal trauma, or neocompression caused by the iatrogenic placement of prosthetic material that compresses the CN VII/VIII complex..$^{16,19}$ Although there are multiple references to the loss of Wave $\mathrm{V}$ and hearing loss, no correlation has been described regarding the value of IPL evaluation during MVD. Interpeak latency is a relatively consistent measurement when compared with absolute latency, which is susceptible to a host of factors (such as age, sex, and intensity of stimulation). ${ }^{2,10}$ Interpeak latency also eliminates individual variation in the magnitude of the latency of Wave I and the latency of Wave V at baseline and at the point of maximal change. The primary aim of this paper is to analyze the changes in IPL during 
MVD and to evaluate if these changes can predict postoperative hearing loss using data collected at University of Pittsburgh Medical Center.

\section{Methods}

\section{Study Design}

We performed a retrospective analysis of consecutive patients who underwent an MVD procedure for HFS between January 2000 and December 2007 at the University of Pittsburgh Medical Center. Patients with clinical HFS confirmed by electromyographic studies and for whom documented pre- and postoperative hearing function and viable intraoperative neurophysiological BAEP data available were selected for the study. Patients with HFS who had poor preoperative hearing function were excluded from the study. The study was approved by the institutional review board for retrospective review of data on human subjects at the University of Pittsburgh Medical Center.

\section{Microvascular Decompression for HFS}

Patients were given short-acting muscle relaxants for intubation; no additional muscle relaxants were administered for the purpose of intraoperative electromyographic monitoring of CN VII in order to evaluate the disappearance of lateral spread as a sign of adequate decompression. ${ }^{6,12}$ Microvascular decompression was performed using a retromastoid craniotomy with the patient in a park bench position, and the patient was maintained on a balance of inhalational and intravenous anesthetic agents. The conflicting vessels were identified, and CN VII was freed by placement of Teflon pledgets. ${ }^{5}$

\section{Perioperative Audiological Investigations and Hearing Loss Criteria}

We considered tone audiometry consisting of PTA and SDSs to be the indicators of auditory function. ${ }^{14,19}$ An otoneurological examination was performed in all patients before surgery (median 1 day, range 1-49 days) and after surgery (median 7 days, range 1-90 days), consisting of an audiogram with measurement of pure tone thresholds (air and bone conduction for octave frequencies $250-8000 \mathrm{~Hz}$ ). Values obtained at 500, 1000, and 2000 $\mathrm{Hz}$ were used to calculate the PTA in decibels. The SDSs were obtained by monitored live voice using phonemically balanced word lists at $40 \mathrm{~dB}$ above the calculated PTA. To avoid misinterpretation of audiometry, postoperative otoscopy and tympanometry were performed to detect conductive hearing loss due to middle ear dysfunction in relation to opening of mastoid cells during craniotomy.

Postoperative hearing loss status was assessed using the 1995 American Academy of Otolaryngology-Head and Neck Surgery classification system. ${ }^{3}$ Class B refers to useful or serviceable hearing loss, and Class C/D refers to nonserviceable hearing loss that is not amenable to hearing aids (Table 1). ${ }^{3}$

\section{Intraoperative Monitoring Recordings}

All patients who received MVD underwent preop-
TABLE 1: American Academy of Otolaryngology-Head and Neck Surgery classification system for hearing loss

\begin{tabular}{ccc}
\hline Hearing Class & PTA $(\mathrm{dB})$ & SDS \% \\
\hline A & $<30$ & $>70$ \\
B & $30-50$ & $>50$ \\
C & $>50$ & $>50$ \\
D & any level & $<50$ \\
\hline
\end{tabular}

erative baseline and continuous intraoperative BAEP monitoring. The right and left ears were stimulated independently, using alternating rarefaction and condensation clicks with at least an 85-dB hearing level. A stimulus rate of $17.5 \mathrm{~Hz}$ was used. White noise was applied to the contralateral ear at the $65-\mathrm{dB}$ hearing level. The observation interval was $12 \mathrm{msec}$. At least 256 responses were averaged to an epoch. The recording electrodes for BAEP monitoring were positioned as follows: Ch1, vertex to left ear mastoid $\mathrm{Cz} / \mathrm{A} 1$; Ch2, vertex to right ear mastoid $\mathrm{Cz} /$ $\mathrm{A} 2$; and $\mathrm{Ch} 3$, vertex to $\mathrm{C}-2$ (Cz/Cv2). The amplifier bandpass was $100-1000 \mathrm{~Hz}$ for all channels. Baseline responses were obtained after anesthesia induction and patient positioning. In addition, lateral spread recordings were made to assay the sufficiency of the MVD of CN VII.

\section{Alarm Criteria}

A decrease in the amplitude of Wave $\mathrm{V}$ by more than $50 \%$ and/or an increase in the latency of Wave $\mathrm{V}$ by $0.5 \mathrm{msec}$ or more (compared with baseline responses) in more than 2 successive trials is considered to be a clinically significant change., ${ }^{4,914,18}$ The primary reason for using more than 2 trials is to identify such changes caused by other issues such as anesthesia or technical problems. To date there are no universally accepted alarm criteria for changes in IPLs of Waves I-III and of Waves I-V during MVD. Physician oversight and interpretation was performed using a combined on-site and remote model used by the University of Pittsburgh Medical Center. ${ }^{20}$

\section{Analysis of Neurophysiological Parameters}

Waves I, III, and V were identified, and the IPLs between Waves I-III, III-V, and I-V were identified for analysis at the following times during the procedure: 1) baseline recording after the patient is intubated and positioned; 2) Change I Start, defined when a first persistent decrease in amplitude of Wave $\mathrm{V}$ greater than $50 \%$ and/or increase in latency of Wave V longer than $0.5 \mathrm{msec}$ compared with baseline is observed; 3) Change Max, defined as the time when a maximum change in the amplitude of Wave $\mathrm{V}$ and/or latency of Wave V compared with baseline (including instances of responses that were completely lost) is observed; and 4) On-Skin change, a final recording at the time of skin closure. If all waveforms were lost (loss of response), amplitude was recorded as 0 and latency was recorded as $12 \mathrm{msec}$, which is the maximum observation interval.

\section{Statistical Analysis}

The prognostic value of intraoperative IPL changes 


\section{Interpeak latency evaluation and microvascular decompression}

was estimated by correlating changes during surgery with pre- and postoperative audiometry and SDSs for any hearing loss. With hearing loss so classified (Table 1), we compared IPL variables between Class A/B and Class C/D. Due to skewed distribution, the statistical significance of differences in the delay of waves was determined using the Kruskal-Wallis test, and the Levene test was used to determine if individual group variances were similar. The chi-square test and Fisher exact test for categorical variables were used to examine hearing outcome for changes in the IPL variables. A binary logistic regression analysis was performed on independent IPL variables of Waves IIII and Waves I-V at Change Max and On-Skin. Further analysis of the same variables was performed adjusting for loss of response (waveform loss). Statistical analysis was calculated using SPSS (version 20, IBM SPSS, Inc.).

\section{Results}

\section{Incidence of Hearing Loss}

Figure 1 displays the outcomes of MVD in our study group. Of 93 patients, $25(26.88 \%)$ had a change in hearing class. Sixteen $(17.20 \%)$ of these patients were classified in Class B and 9 (9.68\%) were classified in Class $\mathrm{C} / \mathrm{D}$ (nonserviceable hearing loss). A loss of response/ waveforms during surgery was observed in 23 patients, of whom 11 recovered during the course of the surgery.

\section{Analysis of IPL Variables in Class A/B Versus Class $C / D$}

Logistic regression analysis of independent IPL variables demonstrated that maximum changes in IPLs of Waves I-III and IPLs of Waves I-V and On-Skin changes IPLs of Waves I-V increases the odds of hearing loss (Table 2). However, on adjusting the same variables for loss of response, IPL variables did not increase the odds of hearing loss (Table 3).

\section{Relationship Between the Latency of Wave V and IPL}

Figure 2 shows the relationship between the latency
TABLE 2: Results of Class $A / B$ versus Class $C / D$ for independent IPL variables

\begin{tabular}{ll}
\hline \multicolumn{1}{c}{ Parameter } & \multicolumn{1}{c}{ OR $(95 \% \mathrm{CI})$} \\
\hline Change Max IPLs of Waves I-III & $1.242(1.008-1.53)$ \\
Change Max IPLs of Waves I-V & $1.496(1.066-2.098)$ \\
On-Skin IPLs of Waves I-III & $1.202(0.949-1.522)$ \\
On-Skin IPLs of Waves I-V & $1.381(1.005-1.898)$ \\
\hline
\end{tabular}

of Wave V and IPLs of Waves I-III, I-V, and III-V for all hearing classes combined at Change Max (no hearing loss and hearing loss). The IPLs of Waves I-V had a strong positive correlation with the latency of Wave V, and the IPLs of Waves I-III and of Waves III-V had a weakly positive and poor correlations, respectively, with the latency of Wave V. Figure 3 shows the comparison of the latency of Wave V and IPLs of Waves I-V for age and sex. Results of the Student t-test analysis of the latency of Wave V and the IPLs of Waves I-V were not significantly different for age and sex.

\section{Discussion}

The vestibulocochlear nerve is susceptible to iatrogenic injury during procedures performed in and around the cerebellopontine angle. Hearing loss in MVD is much more preventable compared with other procedures involving the cerebellopontine angle as the manipulation can be stopped, and persistent hearing deficits can be prevented. Conventionally, the latency and amplitude of Wave $\mathrm{V}$ were the variables used to monitor CN VIII. However, IPL is a relatively consistent variable when compared with the absolute latency of waveforms, which is susceptible to a host of factors (such as stimulus intensity, age, and sex). ${ }^{2,10}$ In the present study, the changes in the IPLs of Waves I-V seemed to strongly reflect absolute latency of Wave V, making them reliable alternative intraoperative measurement and valid alarm criteria.

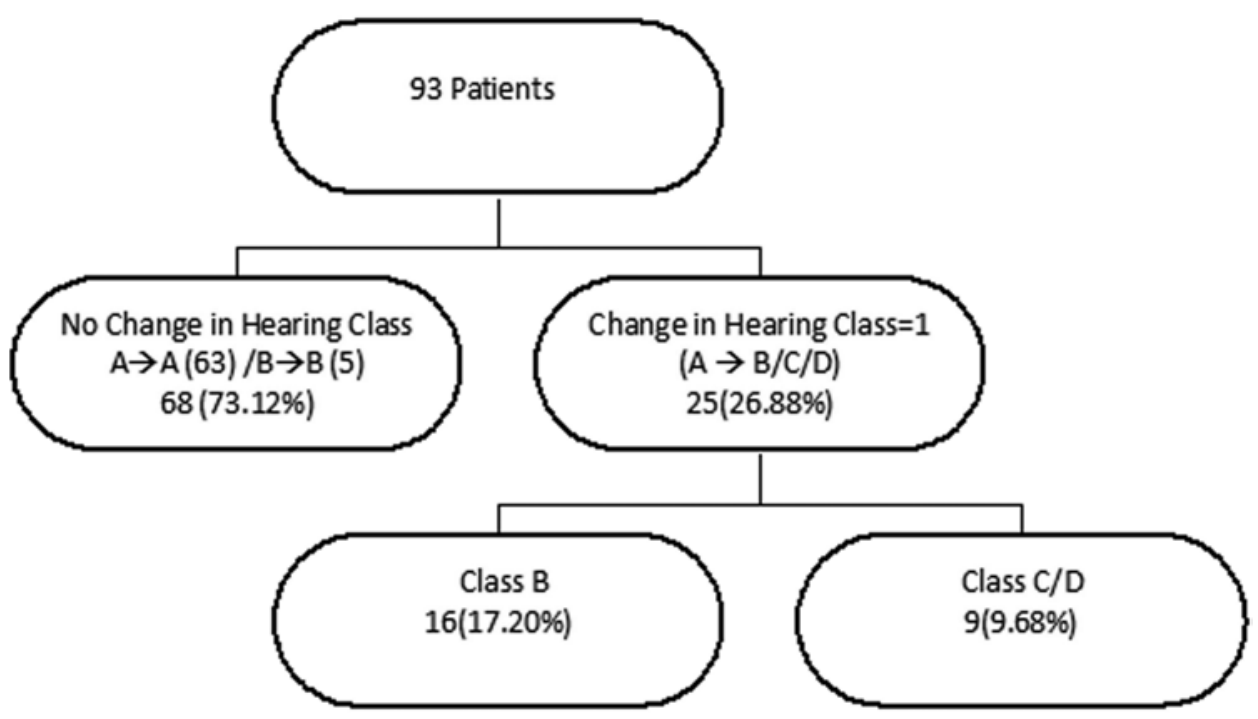

FIG. 1. Chart showing the outcomes of MVD in the study group. 


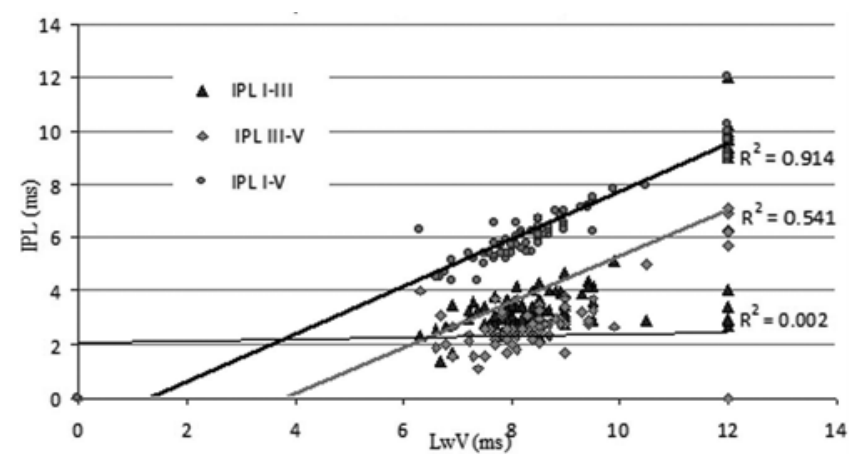

FIG. 2. Relationship between the latency of Wave $\mathrm{V}(\mathrm{LwV})$ and the IPLs of Waves I-III, I-V, and III-V at Change Max (all hearing classes combined).

The IPLs of Waves I-III represent impulse transmission from the distal portion of CN VIII up to the cochlear nucleus. ${ }^{2,8,18}$ As MVD procedures primarily involve manipulation of the CN VIII proper or where it exits, any potential insult to CN VIII should affect impulse conduction across this segment of auditory pathway, which would be reflected as prolongation of the IPLs of Waves I-III., ${ }^{2,718}$ Measurement of Wave III is difficult and unreliable during MVD due to a poor signal-to-noise ratio. The changes in IPLs of Waves I-III can indicate very focal changes in the auditory pathways but due to recording difficulty they are typically unreliable in predicting hearing loss during MVD. The apparent increase in the odds of hearing loss by the IPLs of Waves I-III at Change Max was nullified on adjusting it for loss of response. No significant changes in the IPLs of Waves III-V were noted during MVD procedures in this series. The potentials of IPLs of Waves III-V represent the conduction of auditory impulses in the brainstem. ${ }^{2,8,18}$ In addition, changes in the IPLs of Waves III-V did not correlate well with changes in the latency of Wave V, which makes these IPLs even less valuable during MVD.

The IPLs of Waves I-V reflect total central auditory conduction from the distal part of CN VIII to the inferior
TABLE 3: Results of Class A/B versus Class C/D for IPL variables adjusted for loss of response

\begin{tabular}{lc}
\hline \multicolumn{1}{c}{ Parameter } & OR $(95 \%$ CI) \\
\hline Change Max IPLs of Waves I-III & $1.003(0.762-1.321)$ \\
Change Max IPLs of Waves I-V & $1.028(0.551-1.917)$ \\
On-Skin IPLs of Waves I-III & $1.016(0.766-1.348)$ \\
On-Skin IPLs of Waves I-V & $1.096(0.728-1.651)$ \\
\hline
\end{tabular}

colliculus and include components of the IPLs of Waves I-III and of Waves III-V.,10 The latencies of Waves I and $\mathrm{V}$ can be measured consistently during MVD with a good signal-to-noise ratio. Traction on CN VIII can increase the latency and decrease the amplitude of these waves. Acutely the increase in latency of a waveform is directly proportional to the magnitude of the stress. In nerve stretch studies, a reversible increase in latency and a decrease in amplitude were not associated with ischemic changes in the nerve. The loss of latency and amplitude for prolonged periods of time is associated with poor recovery, ${ }^{22}$ similar to our findings.

Brainstem auditory evoked potential variables to be used as alarm criteria should reliably predict hearing loss during MVD. Although Change Max and On-Skin IPL of Wave I-V changes independently seemed to increase the odds of hearing loss, the results were not consistent after adjusting for loss of response. It is possible that latency changes were present during early retraction of CN VIII and these changes are reversible without hearing loss. However, our current analysis was limited and did not characterize the temporal changes in the latency and amplitude of the BAEP waveforms during MVD. This was a retrospective review of the data, which also limits the amount of information collected and analyzed.

\section{Conclusions}

Changes in the IPLs of Waves I-V correlate with

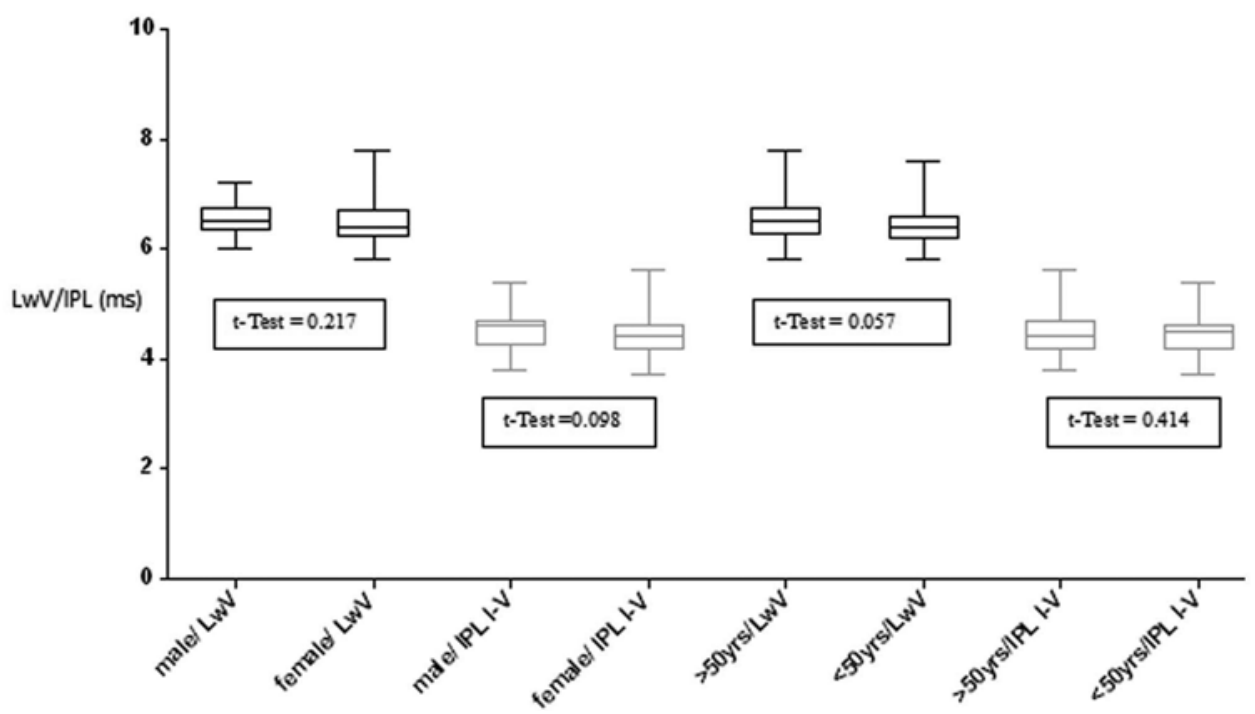

FIG. 3. Comparison of the latency of Wave $V$ and the IPLs of Waves I-V for age ( $<50$ years or $>50$ years) and sex. 


\section{Interpeak latency evaluation and microvascular decompression}

changes in the latency of Wave V during MVD. Changes in the IPLs of Waves I-III and of Waves I-V did not increase the odds of hearing loss after MVD after adjusting for the loss of BAEP waveforms. Further analysis with a combination of variables including changes in latency and amplitude of the BAEPs needs to be conducted before an alarm criterion is adopted.

\section{Disclosure}

The authors report no conflict of interest concerning the materials or methods used in this study or the findings specified in this paper.

Author contributions to the study and manuscript preparation include the following. Conception and design: Thirumala. Acquisition of data: Thirumala. Analysis and interpretation of data: Thirumala, Ilangovan. Drafting the article: Thirumala, Ilangovan. Critically revising the article: Thirumala, Ilangovan. Reviewed submitted version of manuscript: Thirumala. Approved the final version of the manuscript on behalf of all authors: Thirumala. Statistical analysis: Thirumala. Administrative/technical/material support: Thirumala, Balzer, Crammond, Habeych. Study supervision: Thirumala.

\section{References}

1. Barker FG II, Jannetta PJ, Bissonette DJ, Shields PT, Larkins MV, Jho HD: Microvascular decompression for hemifacial spasm. J Neurosurg 82:201-210, 1995

2. Chiappa KH, Hill RA: Brain stem auditory evoked potentials: interpretation, in Chippa KH (ed): Evoked Potentials in Clinical Medicine. Philadelphia: Lippincott-Raven, 1997, pp 199-251

3. Committee on Hearing and Equilibrium: Guidelines for the evaluation of hearing preservation in acoustic neuroma (vestibular schwannoma). Otolaryngol Head Neck Surg 113: 179-180, 1995

4. Hatayama T, Møller AR: Correlation between latency and amplitude of peak $\mathrm{V}$ in the brainstem auditory evoked potentials: intraoperative recordings in microvascular decompression operations. Acta Neurochir (Wien) 140:681-687, 1998

5. Jannetta PJ, Abbasy M, Maroon JC, Ramos FM, Albin MS: Etiology and definitive microsurgical treatment of hemifacial spasm. Operative techniques and results in 47 patients. J Neurosurg 47:321-328, 1977

6. Joo WI, Lee KJ, Park HK, Chough CK, Rha HK: Prognostic value of intra-operative lateral spread response monitoring during microvascular decompression in patients with hemifacial spasm. J Clin Neurosci 15:1335-1339, 2008

7. Legatt AD: Mechanisms of intraoperative brainstem auditory evoked potential changes. J Clin Neurophysiol 19:396-408, 2002

8. Legatt AD, Arezzo JC, Vaughan HG Jr: The anatomic and physiologic bases of brain stem auditory evoked potentials. Neurol Clin 6:681-704, 1988

9. Little JR, Lesser RP, Lueders H, Furlan AJ: Brain stem auditory evoked potentials in posterior circulation surgery. Neurosurgery 12:496-502, 1983

10. Markand ON: Brainstem auditory evoked potentials. J Clin Neurophysiol 11:319-342, 1994

11. Marneffe V, Polo G, Fischer C, Sindou M: [Microsurgical vascular decompression for hemifacial spasm. Follow-up over one year, clinical results and prognostic factors. Study of a series of 100 cases.] Neurochirurgie 49:527-535, 2003 (Fr)

12. Neves DO, Lefaucheur JP, de Andrade DC, Hattou M, Ahdab $\mathrm{R}$, Ayache SS, et al: A reappraisal of the value of lateral spread response monitoring in the treatment of hemifacial spasm by microvascular decompression. J Neurol Neurosurg Psychiatry 80:1375-1380, 2009

13. Park K, Hong SH, Hong SD, Cho YS, Chung WH, Ryu NG: Patterns of hearing loss after microvascular decompression for hemifacial spasm. J Neurol Neurosurg Psychiatry 80: 1165-1167, 2009

14. Polo G, Fischer C, Sindou MP, Marneffe V: Brainstem auditory evoked potential monitoring during microvascular decompression for hemifacial spasm: intraoperative brainstem auditory evoked potential changes and warning values to prevent hearing loss - prospective study in a consecutive series of 84 patients. Neurosurgery 54:97-106, 2004

15. Radtke RA, Erwin CW, Wilkins RH: Intraoperative brainstem auditory evoked potentials: significant decrease in postoperative morbidity. Neurology 39:187-191, 1989

16. Rizvi SS, Goyal RN, Calder HB: Hearing preservation in microvascular decompression for trigeminal neuralgia. Laryngoscope 109:591-594, 1999

17. Samii M, Günther T, Iaconetta G, Muehling M, Vorkapic P, Samii A: Microvascular decompression to treat hemifacial spasm: long-term results for a consecutive series of 143 patients. Neurosurgery 50:712-719, 2002

18. Schramm J, Mokrusch T, Fahlbusch R, Hochstetter A: Detailed analysis of intraoperative changes monitoring brain stem acoustic evoked potentials. Neurosurgery 22:694-702, 1988

19. Sindou MP: Microvascular decompression for primary hemifacial spasm. Importance of intraoperative neurophysiological monitoring. Acta Neurochir (Wien) 147:1019-1026, 2005

20. Thirumala PD, Kassasm AB, Habeych M, Wichman K, Chang YF, Gardner P, et al: Somatosensory evoked potential monitoring during endoscopic endonasal approach to skull base surgery: analysis of observed changes. Neurosurgery 69 (1 Suppl Operative):ons64-ons76, 2011

21. Thirumala PD, Shah AC, Nikonow TN, Habeych ME, Balzer JR, Crammond DJ, et al: Microvascular decompression for hemifacial spasm: evaluating outcome prognosticators including the value of intraoperative lateral spread response monitoring and clinical characteristics in 293 patients. J Clin Neurophysiol 28:56-66, 2011

22. Wall EJ, Massie JB, Kwan MK, Rydevik BL, Myers RR, Garfin SR: Experimental stretch neuropathy. Changes in nerve conduction under tension. J Bone Joint Surg Br 74:126-129, 1992

Manuscript submitted November 19, 2012.

Accepted December 6, 2012.

Portions of the abstract were presented as a poster at the 59th Annual Meeting of the American Association of Neuromuscular \& Electrodiagnostic Medicine, October 3-6, 2012, in Orlando, Florida.

Please include this information when citing this paper: DOI: 10.3171/2012.12.FOCUS12393.

Address correspondence to: Parthasarathy D. Thirumala, M.D., M.S., Center for Clinical Neurophysiology, Department of Neurological Surgery, UPMC Presbyterian, 200 Lothrop Street, Suite B-400, Pittsburgh, Pennsylvania 15213. email: thirumalapd@upmc. edu. 